\title{
6p25 Deletion and Anesthetic Implications During Growing Rod Insertion for Early Onset Scoliosis
}

\author{
Hina Walia ${ }^{\mathrm{a}, \mathrm{d}}$, Mumin Hakim ${ }^{\mathrm{a}}$, Emmett Whitakera, b, Walter Samora ${ }^{\mathrm{c}}$, Jan E. Klamarc, \\ Joseph D. Tobias ${ }^{\mathrm{a}, \mathrm{b}}$
}

\begin{abstract}
$6 \mathrm{p} 25$ is a rare yet clinically identifiable disorder that results from the deletion of the short arm of chromosome 6. Due to the craniofacial, central nervous system, renal, cardiac, and skeletal abnormalities, anesthetic care may be required during various surgical interventions. The associated structural anomalies and end-organ involvement may impact the perioperative care. We present a 4-year-old girl with subtelomeric $6 \mathrm{p} 25$ deletion who required anesthetic care during placement of growing rods to treat progressive scoliosis. A review of literature was performed, the end-organ involvement was discussed, and options for anesthetic care were presented.
\end{abstract}

Keywords: 6p25 deletion; Posterior spinal fusion; Scoliosis

\section{Introduction}

6 p25 is a rare, yet clinically identifiable disorder that results from the deletion of the short arm of chromosome 6. The breakpoints may be at various loci along the sixth chromosome including 6p22.2, 6p23, 6p24.2, and 6p25.1 [1, 2]. Deletions can be categorized as terminal (breakpoints within the 6p24pter region) or interstitial (breakpoints within the 6p22ep24 region) with varying phenotypes resulting depending on the site of breakage and deletion [2-4]. Fluorescence in situ hybridization (FISH) analysis has helped to confirm the location of the deletion with terminal deletions being more common than interstitial deletions or complex rearrangements $[5,6]$.

Manuscript accepted for publication February 02, 2017

aDepartment of Anesthesiology \& Pain Medicine, Nationwide Children's Hospital, Columbus, OH, USA

bepartment of Anesthesiology, The Ohio State University, Columbus, $\mathrm{OH}$, USA

'Department of Orthopedic Surgery, Nationwide Children's Hospital \& The Ohio State University, Columbus, OH, USA

${ }^{\mathrm{d} C}$ Corresponding Author: Hina Walia, Department of Anesthesiology \& Pain Medicine, Nationwide Children's Hospital, 700 Children's Drive, Columbus, OH 43205, USA. Email: Hina.Walia@nationwidechildrens.org

doi: https://doi.org/10.14740/jmc2748w
Commonly associated defects with the interstitial deletions include a short neck, hand abnormalities, structural eye abnormalities, central nervous system (CNS), renal, and cardiac defects while common defects with the terminal deletions may involve ocular involvement of the anterior chamber of the eye (bilateral hyperopia), iris dysplasia, hypoplastic irides, megalocornea, sclerocornea, as well as other end organs resulting in hearing loss, cardiac malformations, hypertelorism, mid-face hypoplasia, and low set ears [7-12]. Developmental delay has been described in all cases.

CNS involvement may include cystic spaces within the periventricular white matter, white matter abnormalities, Dandy-Walker variant, hydrocephalus, cerebellar and posterior fossa malformations including cerebellar vermis hypoplasia, and enlargement of the cisterna magna, and agenesis of the corpus callosum [13-17]. Given the multiple congenital anomalies in various organ systems, anesthetic care may be required during surgical correction or palliation. We present a 4-yearold girl with a sub-telomeric $6 \mathrm{p} 25.3>6 \mathrm{p} 24.3$ deletion who required anesthetic care for placement of growing rods to treat progressive scoliosis. The literature involving these patients was reviewed, end-organ involvement was discussed, and options for anesthetic care were presented.

\section{Case Report}

Institutional Review Board approval is not required at Nationwide Children's Hospital (Columbus, $\mathrm{OH}$ ) for the presentation of a single case report. The patient was a 4-year-old $14.5 \mathrm{~kg}$ young girl who presented for growing rod placement for early onset scoliosis of the thoracolumbar region. Her past medical history was significant for sub-telomeric deletion of $6 \mathrm{p} 25$ with the specific deleted region being $6 \mathrm{p} 25.3>6 \mathrm{p} 24.3$. Birth history included a term birth via cesarean section at an outside hospital. Birth weight was 3,210 g with APGAR scores of 8 and 9. During the immediate neonatal period, a low oxygen saturation $(80-82 \%)$ was noted with the need for supplemental oxygen by nasal cannula as well as a loud murmur on auscultation. She was transferred to our institution for evaluation of hypoxemia, a heart murmur, and abnormal facies. Echocardiogram revealed tetralogy of Fallot (TOF). Chromosomal analysis obtained due to the dysmorphic facies revealed the chromosomal deletion syndrome. Additional past medical history 
included bilateral sensorineural hearing loss for which she was fitted with hearing aids as well as the need for speech and language delay. Her past surgical history was significant for TOF repair at 4 months of age with ventricular septal defect closure, transannular patch, partial pulmonary valvectomy, infundibular resection, and patent foramen ovale closure. Additionally, bilateral ear tube insertion and auditory brainstem response had been performed previously. She was not currently on any home medications and had no known allergies. Preoperative physical examination revealed a young girl in no acute distress with dysmorphic facial features and scoliosis. Airway examination was normal except for a Mallampati grade 3 view. Preoperative laboratory evaluation including electrolytes, renal function, coagulation function, blood glucose and hepatic function was normal. The patient was held nil per os for $8 \mathrm{~h}$ for solids and $2 \mathrm{~h}$ for liquids prior to surgery and was transported to the operating room where routine American Society of Anesthesiologists' monitors were applied. Anesthetic induction was performed with the inhalation of incremental concentrations of sevoflurane in nitrous oxide and oxygen. Bag-valvemask ventilation was provided without difficulty and rocuronium $(0.5 \mathrm{mg} / \mathrm{kg})$ administered to facilitate endotracheal intubation. Direct laryngoscopy was performed with a Miller 2 blade, revealing a Cormack-Lehane grade I view. A $4.5 \mathrm{~mm}$ cuffed endotracheal tube was placed on the first attempt and the cuff slowly inflated to seal the airway at a continuous positive airway pressure (CPAP) of $20 \mathrm{~cm} \mathrm{H}_{2} \mathrm{O}$. Two peripheral venous cannulas and an arterial cannula were placed. Per our usual practice to allow for neurophysiological monitoring during spinal surgery, anesthesia was maintained with desflurane titrated to maintain the bispectral index (BIS) at 50 - 60 and a sufentanil infusion of $0.1-0.3 \mu \mathrm{g} / \mathrm{kg} / \mathrm{h}$ to maintain the mean arterial pressure at $55-70 \mathrm{~mm} \mathrm{Hg}$ [18]. Baseline neurophysiological monitoring including motor-evoked potentials (MEP) and somatosensory-evoked potentials (SSEPs) was obtained. The patient was turned and positioned prone for posterior spinal fusion with Magec $^{\circledR}$ rods from T3-L2 with a total surgical time of $148 \mathrm{~min}$. Hemodynamic status during the surgical procedure was unremarkable without hypotension or clinically significant changes in heart rate. Intraoperative fluids included $430 \mathrm{~mL}$ of Normosol ${ }^{\circledR}$ and $100 \mathrm{~mL}$ of $5 \%$ albumin. No allogeneic blood products were administered. The estimated blood loss was $30 \mathrm{~mL}$. During wound closure, the sufentanil infusion was discontinued. Following completion of the surgical procedure, the patient was turned supine and her trachea was extubated when awake. The patient was transferred to the postanesthesia care unit (PACU) and then admitted to the inpatient surgical ward. Postoperative pain control was provided with hydromorphone $(0.1 \mathrm{mg}$ per dose) delivered via nurse-controlled analgesia (NCA) as well as intravenous acetaminophen and ketorolac. The remainder of her postoperative course was uncomplicated and she was discharged home on postoperative day 4 .

\section{Discussion}

As with other chromosomal abnormalities, deletion of the short arm of chromosome ( $6 \mathrm{p} 25$ deletion) results in a specific pattern of end-organ involvement and anatomical malformations which may require surgical intervention. To date, there are no previous reports regarding the potential anesthetic concerns during the perioperative management of these children. As with the anesthetic care of all patients, the focus of effective perioperative care begins with the preoperative examination and the identification of end-organ involvement related to the chromosomal abnormality.

Given the associated potential for craniofacial involvement including midface hypoplasia, the primary concern for anesthesia providers is the potential for difficulties with airway management and endotracheal intubation [19]. Preoperative airway examination revealed a Mallampati grade 3 airway. Although we were able to successfully accomplish endotracheal intubation using direct laryngoscopy without difficulty, the ability to accomplish adequate bag-valve mask ventilation should be demonstrated prior to the use of neuromuscular blocking agents. Additionally, the appropriate equipment for dealing with a difficult airway should be readily available including equipment for indirect laryngoscopy [20].

Given the potential for associated congenital heart disease, as was noted in our patient, preoperative echocardiography is suggested to evaluate the anatomy, assess myocardial function, the quality of the surgical repair, and the presence of residual shunts or gradients. Reported CNS malformations include Dandy-Walker variant, hydrocephalus, cystic spaces within the periventricular white matter, white matter abnormalities, cerebellar and posterior fossa malformations including cerebellar vermis hypoplasia, enlargement of the cisterna magna, and agenesis of the corpus callosum [13,21]. Preoperative neuroimaging may be indicated and should be guided by the clinical history and physical examination. Associated visual and hearing impairment compounded by developmental delay may impact communication during the perioperative period.

SSEPs and MEPs, used in intraoperative monitoring of spinal cord function, further impact anesthetic management $[22,23]$. MEPs are more sensitive to the choice of anesthetic agents than SSEPs [24-26]. Anesthetic agents both inhaled and intravenous can depress SSEP and MEP with impairment of signal attainment. The effect of the inhaled agent has been shown to be greater than intravenous agents [23]. Per our usual routine, we use 0.5 minimum alveolar concentrations (MAC) of the volatile anesthetic agent, desflurane, supplemented with a benzodiazepine (midazolam) as needed to ensure amnesia with the potent opioid, sufentanil, to provide analgesia and control the hemodynamic response to surgical stimulation. This combination allows for rapid awakening should there be changes in the MEP or SSEP monitoring [18].

Scoliosis may also impact respiratory function, predisposing these patients to perioperative respiratory insufficiency and failure. The goal of surgical intervention is to prevent progression of the scoliosis and its effects on long-term respiratory function [27]. The reduction in lung volumes and the presence of ventilation/perfusion mismatch can be seen in patients when the Cobb angle (lateral curvature) exceeds $65^{\circ}$. Pulmonary hypertension and right ventricular hypertrophy may develop in severe cases $\left(\mathrm{Cobb}\right.$ angle $>100^{\circ}$ ) [28]. Following scoliosis surgery, including patients with idiopathic scoliosis without co-morbid conditions, there is a $40 \%$ decrease in vital capacity 
during the postoperative period [28]. Postoperative respiratory dysfunction may be greater in patients with associated comorbid conditions involving the CNS, cardiac and respiratory systems, leading to the need for prolonged respiratory support. These issues can be lessened by preoperative optimization of pulmonary function and the use of non-invasive ventilatory support $[29,30]$. Although needed for the control of pain, postoperative opioid administration may impact central control of ventilation and further compromise perioperative respiratory function. Adjunctive agents (acetaminophen or non-steroidal anti-inflammatory agents) may decrease opioid requirements and their associated adverse effects. In our patient, postoperative analgesia was provided by nurse-controlled analgesia using hydromorphone and the use of adjunctive agents (intravenous acetaminophen and ketorolac).

In summary, we present a patient with 6 p25 deletion, a recognized chromosomal deletion syndrome, characterized by a specific spectrum of congenital abnormalities. No previous publication has reviewed the anesthetic implications of this disorder. Specific perioperative co-morbid concerns include craniofacial involvement which may impact airway management, associated CHD, and CNS involvement. These concerns may be magnified as was the case in our patient by the specific surgical procedure involved. In our patient, who was scheduled for placement of growing rods to treat scoliosis, additional concerns included anesthetic management to maximize MEP and SSEP monitoring as well as the impact of scoliosis and the surgical procedure on postoperative respiratory function.

\section{References}

1. Alashari M, Chen E, Poskanzer L. Partial deletion of chromosome 6p: autopsy findings in a premature infant and review of the literature. Pediatr Pathol Lab Med. 1995;15(6):941-947.

2. Davies AF, Mirza G, Sekhon G, Turnpenny P, Leroy F, Speleman F, Law C, et al. Delineation of two distinct $6 \mathrm{p}$ deletion syndromes. Hum Genet. 1999;104(1):64-72.

3. Palmer CG, Bader P, Slovak ML, Comings DE, Pettenati MJ. Partial deletion of chromosome 6p: delineation of the syndrome. Am J Med Genet. 1991;39(2):155-160.

4. Davies AF, Olavesen MG, Stephens RJ, Davidson R, Delneste D, Van Regemorter N, Vamos E, et al. A detailed investigation of two cases exhibiting characteristics of the 6 deletion syndrome. Hum Genet. 1996;98(4):454-459.

5. Bedoyan JK, Lesperance MM, Ackley T, Iyer RK, Innis JW, Misra VK. A complex 6p25 rearrangement in a child with multiple epiphyseal dysplasia. Am J Med Genet A. 2011;155A(1):154-163.

6. Misceo D, Bjorgo K, Ormerod E, Ringen O, Rocchi M, van der Hagen CB, Frengen E. A de novo $6 p$ interstitial deletion and a complex translocation involving chromosomes 2, 6, and 14 in a mildly developmentally delayed patient. Am J Med Genet A. 2008;146A(24):3230-3233.

7. Anderlid BM, Schoumans J, Hallqvist A, Stahl Y, Wallin A, Blennow E, Nordenskjold M. Cryptic subtelomeric $6 \mathrm{p}$ deletion in a girl with congenital malformations and severe language impairment. Eur J Hum Genet. 2003;11(1):89-92.

8. Davies AF, Mirza G, Flinter F, Ragoussis J. An interstitial deletion of 6p24-p25 proximal to the FKHL7 locus and including AP-2alpha that affects anterior eye chamber development. J Med Genet. 1999;36(9):708-710.

9. Le Caignec C, Swillen A, Van Asche E, Fryns JP, Vermeesch JR. Interstitial $6 \mathrm{q}$ deletion: clinical and array CGH characterisation of a new patient. Eur J Med Genet. 2005;48(3):339-345.

10. Martinez-Glez V, Lorda-Sanchez I, Ramirez JM, RuizBarnes P, Rodriguez de Alba M, Diego-Alvarez D, Ramos $\mathrm{C}$, et al. Clinical presentation of a variant of Axenfeld-Rieger syndrome associated with subtelomeric $6 \mathrm{p}$ deletion. Eur J Med Genet. 2007;50(2):120-127.

11. Piccione M, Antona R, Salzano E, Cavani S, Malacarne M, Morreale Bubella R, Pierluigi M, et al. Array-CGH and clinical characterization in a patient with subtelomeric $6 \mathrm{p}$ deletion without ocular dysgenesis. Am J Med Genet A. 2012;158A(1):150-154.

12. Tonoki H, Harada N, Shimokawa O, Yosozumi A, Monzaki K, Satoh K, Kosaki R, et al. Axenfeld-Rieger anomaly and Axenfeld-Rieger syndrome: clinical, molecularcytogenetic, and DNA array analyses of three patients with chromosomal defects at $6 \mathrm{p} 25$. Am J Med Genet A. 2011;155A(12):2925-2932.

13. Lin RJ, Cherry AM, Chen KC, Lyons M, Hoyme HE, Hudgins L. Terminal deletion of $6 p$ results in a recognizable phenotype. Am J Med Genet A. 2005;136(2):162-168.

14. Chen KM, Cherry AM, Hahn JS, Enns GM. Mild developmental delay in terminal chromosome $6 \mathrm{p}$ deletion. Am J Med Genet A. 2004;129A(2):201-205.

15. van der Knaap MS, Kriek M, Overweg-Plandsoen WC, Hansson KB, Madan K, Starreveld JS, Schotman-Schram $\mathrm{P}$, et al. Cerebral white matter abnormalities in 6p25 deletion syndrome. AJNR Am J Neuroradiol. 2006;27(3):586588.

16. Aldinger AK, Lehmann OJ, Hudgins L, Chizhikov VV, Bassuk AG, Ades LC, Krantz ID, et al. FOXC1 is required for normal cerebellar development and is a major contributor to chromosome 6p25.3 Dandy-Walker malformation. Nat Genet. 2009;41:1037-1042.

17. DeScipio C. The 6p subtelomere deletion syndrome. Am J Med Genet C Semin Med Genet. 2007;145:377-382.

18. Martin DP, Bhalla T, Thung A, Rice J, Beebe A, Samora W, Klamar J, et al. A preliminary study of volatile agents or total intravenous anesthesia for neurophysiological monitoring during posterior spinal fusion in adolescents with idiopathic scoliosis. Spine (Phila Pa 1976). 2014;39(22):E1318-1324.

19. Butler MG, Hayes BG, Hathaway MM, Begleiter ML. Specific genetic diseases at risk for sedation/anesthesia complications. Anesth Analg. 2000;91(4):837-855.

20. Engelhardt T, Weiss M. A child with a difficult airway: what do I do next? Curr Opin Anaesthesiol. 2012;25(3):326-332.

21. DeScipio C. The $6 \mathrm{p}$ subtelomere deletion syndrome. Am J Med Genet C Semin Med Genet. 2007;145C(4):377382. 
22. Deiner S. Highlights of anesthetic considerations for intraoperative neuromonitoring. Semin Cardiothorac Vasc Anesth. 2010;14(1):51-53.

23. Sloan TB, Heyer EJ. Anesthesia for intraoperative neurophysiologic monitoring of the spinal cord. J Clin Neurophysiol. 2002;19(5):430-443.

24. Balvin MJ, Song KM, Slimp JC. Effects of anesthetic regimens and other confounding factors affecting the interpretation of motor evoked potentials during pediatric spine surgery. Am J Electroneurodiagnostic Technol. 2010;50(3):219-244.

25. Reinacher PC, Priebe HJ, Blumrich W, Zentner J, Scheufler KM. The effects of stimulation pattern and sevoflurane concentration on intraoperative motor-evoked potentials. Anesth Analg. 2006;102(3):888-895.

26. Lo YL, Dan YF, Tan YE, Nurjannah S, Tan SB, Tan CT,
Raman S. Intra-operative monitoring in scoliosis surgery with multi-pulse cortical stimuli and desflurane anesthesia. Spinal Cord. 2004;42(6):342-345.

27. Koumbourlis AC. Scoliosis and the respiratory system. Paediatr Respir Rev. 2006;7(2):152-160.

28. Vedantam R, Lenke LG, Bridwell KH, Haas J, Linville DA. A prospective evaluation of pulmonary function in patients with adolescent idiopathic scoliosis relative to the surgical approach used for spinal arthrodesis. Spine (Phila Pa 1976). 2000;25(1):82-90.

29. Jaber S, Michelet P, Chanques G. Role of non-invasive ventilation (NIV) in the perioperative period. Best Pract Res Clin Anaesthesiol. 2010;24(2):253-265.

30. Pelosi P, Jaber S. Non-invasive respiratory support in the perioperative period. Curr Opin Anaesthesiol. 2010;3:2228-2233. 